\section{A l'étonnement général ...}

Tout le monde se mêle de la politique de santé. Après nos sept sages, les partis politiques, les assureurs et autres philosophes de la santé, voici enfin le Crédit Suisse qui nous propose ses recettes pour gérer les coûts de la santé [1]. Nous n'attendions plus que cela. Il est vrai qu'une des plus prestigieuses institutions de notre pays ne pouvait être absente de ce débat, il était temps qu'elle prenne enfin ses responsabilités. Du moment que la Banque Mondiale se prête à sortir l'humanité de la pauvreté, que le Fonds Monétaire Internationale se porte au secours des pays en banqueroute et que Kaspar Villiger nous offre une nouvelle compagnie aérienne, il aurait été absurde que nos banques n'accourent pas au chevet du plus grand de nos malades, de notre système de santé.

Imaginez un seul instant ce qui se passerait dans le cas contraire: Un désintérêt des banques provoquerait une vague de méfiance par rapport au système de santé. Un peu comme à la bourse quand les investisseurs se retirent, on commencerait à douter de la capacité de produire des prestataires de soins. Sentant le crash imminent, les investisseurs se détourneraient rapidement de leurs engagements, les caisses-maladies se tourneraient vers d'autres marchés, en attendant des temps meilleurs et laissant aux médecins le soin de se débrouiller. Les politiques ne voyant plus dans la santé une affaire de gros sous s'occuperaient de nouveau de choses sérieuses comme l'Armée ou l'Agriculture: voilà des secteurs avec des budgets dignes des hommes politiques de niveau!

Mais alors, que se passerait-il avec nos malades? Augmentation dramatique de la mortalité générale, recrudescence de maladies qu'on croyait disparues? Les hôpitaux tomberaient en ruines? Les cabinets médicaux se vendraient à des salons de beauté ou de coiffure?
A l'étonnement général, rien de tout cela se produirait. Les hôpitaux continueraient à prendre les urgences, les chirurgiens, ne sachant de toute façon rien faire d'autre qu'opérer, continueraient d'opérer, de même pour tous les autres spécialistes. Les généralistes eux-aussi continueraient de suivre leurs malades, car auraient-ils une raison de plutôt rester à la maison en tournant les pouces? Et croyez-vous un seul instant que les malades cesseraient de rembourser les prestations? Ils continueraient de payer comme ils l'ont toujours fait. Très vite, de nouvelles formes de solidarité émergeraient faisant preuve d'ingéniosité en matière d'assurance insoupçonnée jusqu'alors. Bref, la médecine resterait la médecine et le monde continuerait, malgré tout, de tourner.

Quelles leçons pouvons nous tirer de cette petite rêverie? Personne peut nous empêcher d'imaginer l'impossible, et de là il n'y plus qu'un pas pour changer les choses qu'on croit immuables. Seul inconvénient: ce concept est révolutionnaire, et comme on sait, notre Constitution ne prévoit pas ce genre de chose. Mais tout de même, si les médecins de ce pays arrivaient tant soit peu à créer quelques propositions originales au lieu de seulement réagir à toutes les sauces auxquelles on nous sert, ce serait déjà un grand progrès face à la situation actuelle.

Courage!

Dr Markus Trutmann

1 Crédit Suisse. Das Schweizer Gesundheitswesen Diagnose für einen Patienten. Economic Briefing Nr. 30. www.credit-suisse.ch. 